\title{
Biomimetic Supramolecular Polymer Networks Exhibiting both Toughness and Self-recovery ${ }^{* *}$
}

\author{
Ji Liu, ${ }^{1}$ Cindy Soo Yun Tan, ${ }^{1,2}$ Ziyi Yu, ${ }^{3}$ Yang Lan, ${ }^{1}$ Chris Abell, ${ }^{3}$ Oren A. Scherman ${ }^{1} * *$
}

In contrast to biological systems that can spontaneously self-repair and adapt to environmental stimuli, most synthetic materials do not display neither self-repair following mechanical deformation nor dynamic adaptiveness. ${ }^{[1,2]}$ The emerging field of "biomimicry", aiming to imitate such natural functions, will enable the construction of hierarchical structures and new materials with improved durability and functionalities. New sophisticated analytical techniques offer scientists an unprecedented understanding of molecular and structural mechanisms, which are involved in the adaptability, stimuli-responsiveness and mechanical integrity found in biological materials and complex systems. ${ }^{[3,4]}$ Moreover, advancement of synthetic strategies, as well as hierarchical structural design and fabrication, allows for the facile construction of high-fidelity structures, materials and high-performance biomimetic systems. ${ }^{[1,5,6]}$

In nature, many structural proteins are essential for specific biological functions and indispensable components to fulfil precise biological roles. ${ }^{[7,8]}$ A representative example is the skeletal muscle protein titin ( $3 \mathrm{MDa}, c a .1 \mathrm{~mm}$ long), which dominates the mechanical strength and toughness of muscles. ${ }^{[9-12]}$ The ability of titin to absorb energy, by force-induced rupture of secondary intramolecular interactions, has been revealed by single molecule force spectroscopy studies. ${ }^{[9,10]}$ Moreover, titin's unfolding process is followed by refolding-induced shape recovery, making it an intriguing design model for artificial adaptive materials. ${ }^{[13,14]}$ Only a limited number of protein- and polypeptidebased materials have been reported in the literatures, following this unfolding/refolding model. ${ }^{[15-17]}$ Guan et al. first reported a biomimetic system incorporating supramolecular quadruple hydrogen bonding moieties, 2-ureido- $4[1 \mathrm{H}]$ -

\footnotetext{
** J.L. is financially supported by the Marie Curie FP7 SASSYPOL ITN (607602) program. C.S.Y.T. thanks the Ministry of Education of Malaysia and Universiti Teknologi MARA for their financial supports. Z.Y. is funded by the EPSRC, Institutional Sponsorship 2012-University of Cambridge EP/K503496/1 and the Translational Grant EP/ H046593/1. O.A.S thanks the EPRSC (EP/F0355351 and EP/G060649/1) and the ERC (ASPiRe, 240629) for their funding.

* 1 Melville Laboratory for Polymer Synthesis, Department of Chemistry, University of Cambridge, Cambridge, CB2 1EW, United Kingdom. E-mail: oas23@cam.ac.uk.

${ }^{2}$ Faculty of Applied Sciences, Universiti Teknologi MARA, 94300 Kota Samarahan, Sarawak, Malaysia.

${ }^{3}$ Department of Chemistry, University of Cambridge, Lensfield Road, Cambridge CB2 1EW, United Kingdom.
}

Supporting information for this article is available on the WWW under or from the author pyrimidones (UPy)s, as modular units, mimicking the immunoglobulin (Ig)-like domains. ${ }^{[18-20]}$ UPy-based materials, however, can be challenging to be incorporated into aqueous systems unless segmented amphiphilic UPy-tethered polymers are used, thus pre-formed fibres in aqueous solution are able to physically cross-link and generate transient supramolecular networks. ${ }^{[21,22]}$

Herein, we present a new class of aqueous dual networks, which incorporate both a small amount of noncovalent cucurbit $[n]$ uril $(\mathrm{CB}[n])$-mediated host-guest interactions $(2.5 \mathrm{~mol} \%)$ and a trace amount of covalent crosslinking $(0.05 \mathrm{~mol} \%)$. The covalent chemical crosslinks serve to maintain the shape of dual network and impart it with elasticity. Supramolecular host-guest interactions, on the other hand, contribute to several mechanical functions simultaneously: (1) they enhance fracture resistance through the rupture of host-guest complexes as sacrificial bonds, thereby toughening the overall network; (2) they enhance fatigue resistance and self-recovery via the reformation of host-guest complexes; and (3) they enhance energy dissipation through the dynamic dissociation/re-association of $\mathrm{CB}$ [8] host-guest complexes. Accordingly, this dual network (Figure 1), consisting of covalent crosslinking as well as dynamic supramolecular host-guest interactions, closely mimics titin's structure/function model.

A universal design strategy, affording the desired material properties, involves both "bottom-up" synthesis and hierarchical construction across multiple length scales. As a supramolecular binding motif, the $\mathrm{CB}[n]$-mediated dynamic host-guest interactions have led to the fabrication of supramolecular polymers, ${ }^{[23]}$ supramolecular polymer networks, ${ }^{[24,25]}$ and other complex systems ranging from molecular engineering to macroscopic functional materials. ${ }^{[26]}$ Herein, a polymerisable guest (1-benzyl-3vinylimidazolium), serving as a non-covalent supramolecular crosslinker upon complexation with $\mathrm{CB}[8]$ in a $2: 1$ manner $\left(K_{a 1}=4.21 \times 10^{7} \mathrm{M}^{-1}, K_{a 2}=4.25 \times 10^{5} \mathrm{M}^{-1}\right.$, see ESI Figure S1), is polymerised with a trace amount of chemical crosslinker $N, N^{\prime}$-methylenebisacrylamide (MBA, 2 mol.\% of the non-covalent CB[8] crosslinks) and a hydrophilic monomer acrylamide, yielding an aqueous dual network (Figure 1a). The CB[8] host-guest complexes form dynamic 'loops' along the polymer chains between two covalent crosslinking sites (Figure 1c). Rupture of the 2:1 guest/CB[8] complexes requires $7.6 \mathrm{kcal} \mathrm{mol}^{-1}\left(\Delta \mathrm{H}_{2}\right.$, see ESI Figure S1), which is a little bit higher but still comparable to the protein unfolding energy $\left(c a .4 \mathrm{~kJ} \mathrm{~mol}^{-1}\right.$ for an 


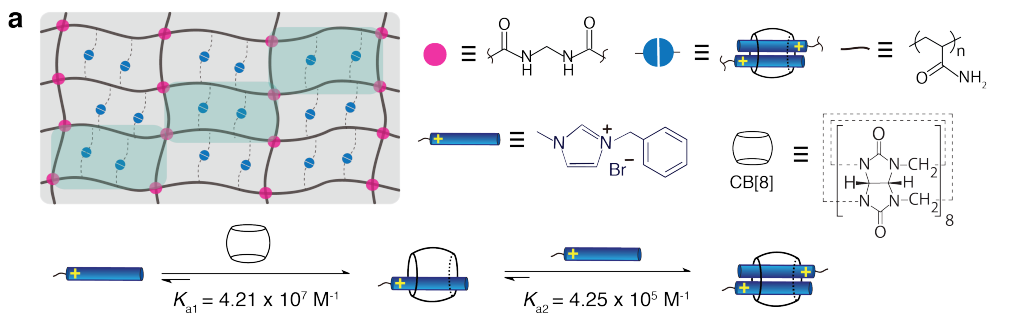

b

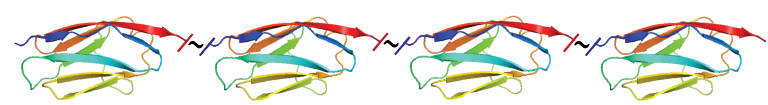

C

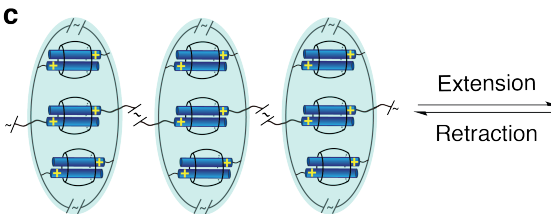

Reformation of host-guest complexes via relaxation

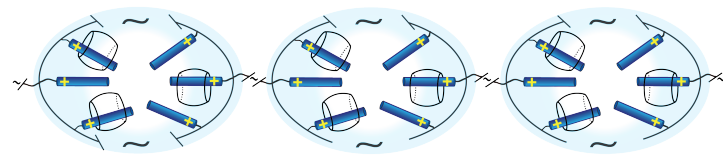

Force-induced dissociation of host-guest complexes

Figure 1. The concept of biomimetic supramolecular network design: a) schematic illustration of a modular dual network composed of non-covalent supramolecular host-guest interactions (blue circles) and chemical crosslinks (pink circles), and the stepwise CB[8] ternary complexation in a 2:1 stoichiometric ratio. b) A typical modular section of titin structure, which has 200-300 repeating immunoglobulin (Ig)-like domains, and modular tertiary-foldeddomain protein that provides the core functionality for hierarchically assembled muscle machinery. c) Schematic illustration of a dual network containing multiple loops held by the $\mathrm{CB}[8]$ host-guest complexation (structure extracted from the shadow area in a), the mechanically-induced dissociation of hostguest complexes, fundamental mechanism for energy dissipation, as well as reformation of the host-guest complexes upon unloading and relaxation. d) Photographs of a strip of the unnotched dual network sample (dimension: $40 \mathrm{~mm}(L) \times 1 \mathrm{~mm}(W) \times 1.5 \mathrm{~mm}(T)$ ) upon stretching to a strain of $17 \times$, e) and a notched sample (notch size $20 \mathrm{~mm})$ to a strain of $7 \times$. f) Image demonstrating the capability of a dual network sample $(40 \mathrm{~mm}(L) \times 2 \mathrm{~mm}(W) \times 1.5 \mathrm{~mm}(T))$ sustaining a weight of $200 \mathrm{~g}$.

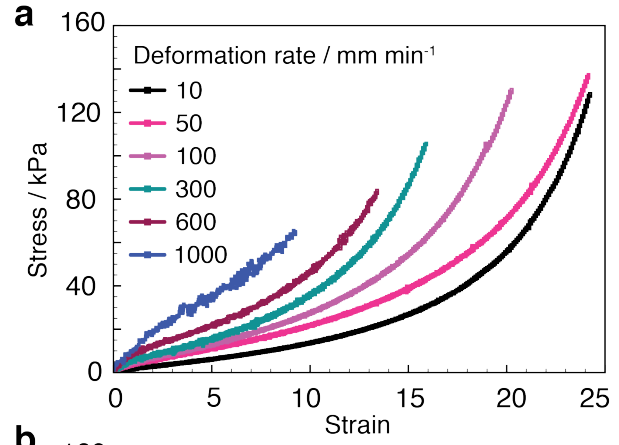

b

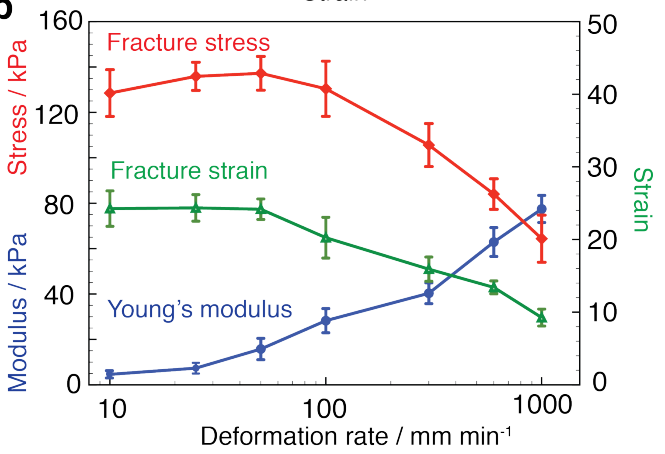

Figure 2. a) Uniaxial tensile behaviour of the supramolecular dual network (dumbbell shape) with different initial deformation rates, ranging from 10 to $1000 \mathrm{~mm} \mathrm{~min}^{-1}$, with nominal stress plotting against strain. b) Deformation rate dependence of fracture stress, fracture strain and Young's Modulus.

immunoglobulin domain). ${ }^{[27]}$ These dual networks could be readily prepared at room temperature in aqueous media upon photo-initiated polymerisation, with a high level of transparency, and they are both tough and durable upon compression (see ESI Figure S2). Besides, the dual network sample exhibits remarkable mechanical strength, which is strong enough to hold $500 \times$ its own weight (Figure 1f) without failure. Control networks prepared without $\mathrm{CB}[8]$ or in the presence of its smaller homologue $\mathrm{CB}$ [7] exhibit moduli that are 2-3 orders of magnitude lower (see ESI Figure S4 and S5), confirming the key role of $\mathrm{CB}[8]$ host-guest interactions to the dual networks' strength. Interestingly, the network rapidly shrinks isotropically without undergoing any cracking at the surface upon freeze drying; furthermore, it can readily swell by absorbing up to $100 \times$ its own weight of water (see ESI Figure S2d).

Uniaxial stretching studies were conducted to further investigate the nonlinear and viscoelastic behaviour of the dual networks (Figure 2a). This dual network exhibits a high stretchability up to $24 \times$ its original length before fracture. Moreover, during the stretching the network sample maintains excellent transparency (Figure 1d) in the visible light range (400-700 nm, see ESI Figure S3). The notchinsensitiveness is evidenced when a notched sample (half of the overall width) was stretched, the notch was dramatically blunted and remained stable during stretching until a critical strain of $7 \times($ Figure 1e). Notably, the Young's modulus increases by $17 \times$ from $4.6 \mathrm{kPa}\left(10 \mathrm{~mm} \mathrm{~min}^{-1}\right)$ to $77.5 \mathrm{kPa}$ $\left(1000 \mathrm{~mm} \mathrm{~min}^{-1}\right)$. Both fracture stress and strain change slightly at low deformation rates (10 to $50 \mathrm{~mm} \mathrm{~min}^{-1}$ ), how- 
a b

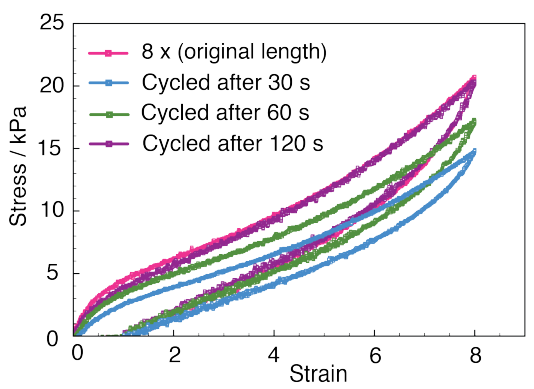

d

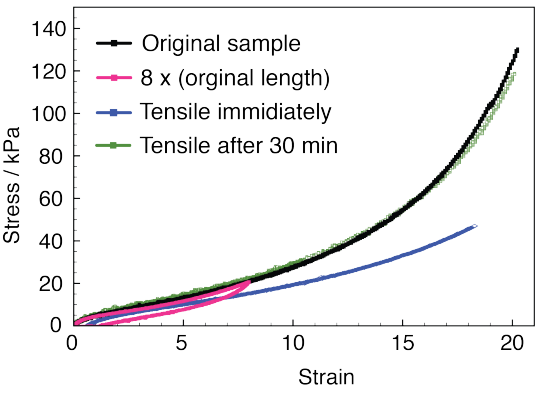

Figure 3. a) Dual network samples were subjected to a loading/unloading cycle of varying strains (deformation rate of $100 \mathrm{~mm} \mathrm{~min}^{-1}$ ). b) Recovery of the network performed by consecutive cyclic tensile tests at a strain of $8 \times$ with different waiting times. c) Dependence of the hysteresis ratio (area ratio of the following hysteresis loop to the first cyclic run) and residual strain on the waiting time. d) Tensile test of a sample immediately (blue curve) after the first loading/unloading cycle strain of $8 \times$ (pink curve), and the self-repaired sample was allowed to sit at room temperature for 30 min (green curve). The high-level of overlap between the original sample curve (black curve) and the self-repaired sample after 30 min (green curve) indicates fast self-recovery of mechanical properties via the reformation of supramolecular host-guest complexes.

ever, decrease at higher rates afterwards. This can be explained by the dynamic dissociation and re-association of the $\mathrm{CB}[8]$ host-guest coingmplexes during the low-rate stretching, which result in the relaxation of overstretched chains under the stress, further maintaing the fracture stress and strain, thus a high strain-at-break detected. ${ }^{[28-31]}$ At higher stretching rates $\left(>100 \mathrm{~mm} \mathrm{~min}^{-1}\right)$, however, the dual network looses its toughness presumably since the host-guest complexes do not have sufficient time to reform after dissociation at time scale of the mechanical deformation, leading to stress propagating at the crack tip. These viscoelastic characters of the $\mathrm{CB}[8]$ dual networks are similar to polyampholyte hydrogels reported by Gong, ${ }^{[30,32-34]}$ alginate hydrogels by Suo, ${ }^{[29]}$ and $\mathrm{Fe}^{3+}$-acrylic acid hydrogels by Zhou, ${ }^{[35]}$ to cite only a few. Even though the modulus is not directly comparable to those previously reported supramolecular hydrogel systems, ${ }^{[29,30,32-36]}$ it is surprising that a small amount of supramolecular crosslinking $(2.5 \mathrm{~mol} \%)$ and trace amount of covalent crosslinking $(0.05 \mathrm{~mol} \%)$, along with an overall mass fraction of only $10 w t \%$, could effectively impart the dual network with remarkable stretchability and strength. Compared with $\mathrm{CB}[n]$-based single networks ${ }^{[25,26]}$ and dual networks (CB[8] and DNA) ${ }^{[24]}$ we have previously reported, which exploited post-polymerisation modification and mixing of guest-bearing polymers and $\mathrm{CB}[8]$, this in situ polymerisation route could overcome inherent challenges, including scale-up and manipulation, scope of starting materials, high viscosity of polymer solution, phase seperation, etc. Dual networks with a variable amount of
$\mathrm{CB}[8]$ crosslinking and covalent crosslinking were also studied (see ESI Figure S6); it was found that an increase in covalent crosslinking could substantially increase the elasticity, while an increase in non-covalent $\mathrm{CB}[8]$ crosslinking could, on the contrary, increase the viscoelasticity of the dual networks. Thus, this synthetic strategy also allows flexibility in tuning the chemical composition, ranging from guest molecule modalities, host/guest complex fraction, covalent/non-covalent crosslinking ratio, overall mass fraction to hydrophilic backbones, thus rationally leading to the development of a full library of $\mathrm{CB}$ [8] dual networks with desirable mechanical properties.

Cyclic tensile tests were carried out to investigate the self-recovery, on account of the dynamic essence of $\mathrm{CB}$ [8] host-guest interactions, similar to the reversible protein folding/unfolding within titin. Stretching-retraction cycles at different strains are shown in Figure 3a, demonstrating significant quasi-plastic deformation and pronounced hysteresis, for which the supramolecular interactions play a critical role. This confirms that force-induced rupture of host-guest complexes as sacrificial bonds contributes to effective energy dissipation, similar to other reported high-toughness networks. ${ }^{[29-35]}$ Through a quantitative measurement, a toughness of $750 \pm 40 \mathrm{~J} \mathrm{~m}^{-2}$ was estimated from a RivlinThomas pure shear test (see ESI Figure S11b), comparable to the toughness of cartilage $\left(1000 \mathrm{~J} \mathrm{~m}^{-2}\right),{ }^{[29]}$ promising potential use as structural biomaterials for regenerative tissue engineering. During the retraction process the residual strain gradually decreases (Figure 3b), which can be as- 


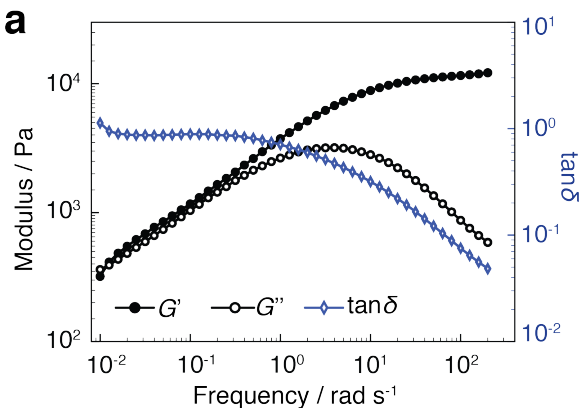

C

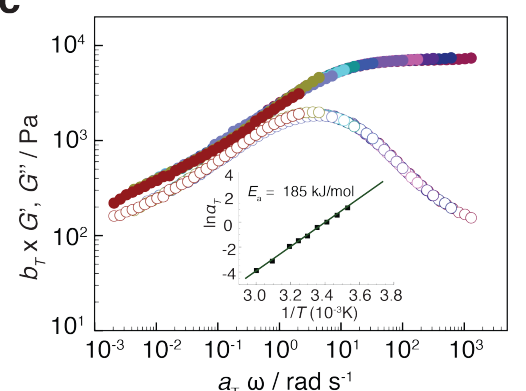

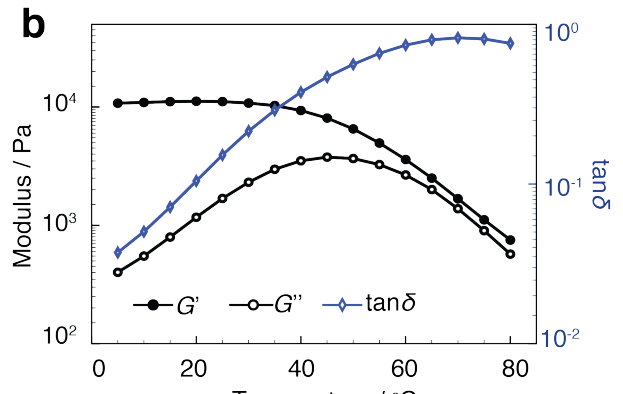

d

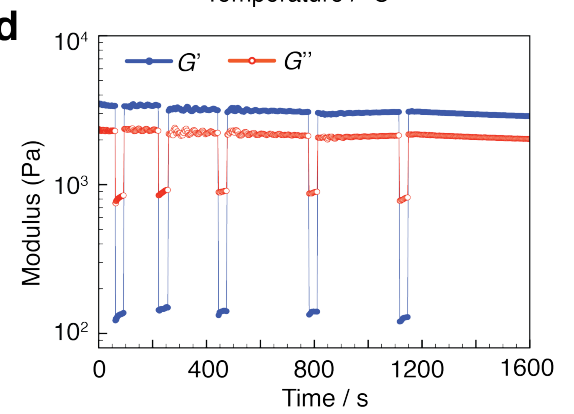

Figure 4. a) $G^{\prime}, G^{\prime \prime}$ and $\tan \theta$ values of the dual network from a dynamic room-temperature frequency sweep (from 0.01 to 200 rad s ${ }^{-1}, 0.5 \%$ strain. b) Temperature sweep (from 5 to $80{ }^{\circ} \mathrm{C}$ with an interval of $5{ }^{\circ} \mathrm{C}, 60 \mathrm{rad} \mathrm{s}^{-1}, 0.5 \%$ strain). c) Master curve of the dual network sample (from 0 to $60{ }^{\circ} \mathrm{C}, 0.5$ $\%$ strain, $10 \mathrm{rad} \mathrm{s}^{-1}$ ). d) Continuous step-strain measurements of the sample at $40{ }^{\circ} \mathrm{C}$ (high-amplitude oscillatory parameters: $\gamma=500 \%, \omega=10$ rad s ${ }^{-1}$; low-amplitude oscillatory parameters: $\gamma=0.5 \%, \omega=10 \mathrm{rad} \mathrm{s}^{-1}$ ).

cribed to the elasticity arising from the covalent crosslinking and chain entanglement, and also the reformation of hostguest complexes. A fifty-percent hysteresis ratio is observed within $5 \mathrm{~s}$ (Figure 3c), while the remaining 50\% recovers following a much slower and complex process. If a waiting time of $120 \mathrm{~s}$ is applied between two consecutive cycles, the network does not display any noticeable plastic deformation, thus demonstrating high fatigue resistance. Similar to the polyampholyte system reported by Gong et al. ${ }^{[30]}$ a twostage recovery process might also be involved during this stress-free recovery. The elasticity arising from chemical crosslinking and chain entanglement dominates the recovery through elastic retraction and association of the previouslycleaved host-guest complexes, leading to faster recovery during the unloading process (strain $>1$ ). When it decreases below a value of 1 , a re-organisation of the re-formed hostguest complexes under the elastic contraction of the primary network leads to complete self-recovery. This was further corroborated by the even faster self-recovery of the dual network with higher covalent crosslinking (CB[8] 2.5 mol.\%, MBA $0.5 \mathrm{~mol} . \%$ ), which exhibits virtually no difference in hysteresis between two consecutive cyclic tests (see ESI Figure S8).

The fast self-recovery and high toughness are also demonstrated in compression with a blade (see ESI Figure S2b), which is followed with a prompt recovery to its original shape after removal of the blade test (see ESI Supporting Movie 1). In order to further illustrate the dual network's ability to dissipate energy, a drop-ball test was performed. When a metal ball $(16 \mathrm{~g}$, diameter of $2 \mathrm{~cm}$, drop-height of $45 \mathrm{~cm}$ ) was dropped onto a network film, a large and recov- erable deformation was observed. Upon hitting the film, the ball stretched it extensively before bouncing back (see ESI Supporting Movie 2). While the film remained intact, vibrated and recovered to its initial flat configuration after successfully dissipating the energy. However, fracture of the covalent network (MBA 2.55 mol. \%) was observed (see ESI Supporting Movie 3). Self-recovery of the dual network sample was then studied with the tensile test. After the sample was subjected to a cyclic strain of $8 \times$, it is marginally weaker than the original material, however, it self-repaired after a 30 min waiting time at room temperature (Figure 3d). As previously reported by Gong et al., ${ }^{[34]}$ the presence of covalent crosslinking dramatically inhibits the self-healing character of a dual network material, here macroscopic selfhealing was also not observed unless all covalent crosslinks were removed from the system.

The dual network exhibits a broad linear viscoelastic regime, typical of a tough hydrogel as depicted in Figure $\mathbf{S 4}$, with a critical yield strain of $80 \%$, above which the network starts to rupture $\left(G^{\prime \prime}>G^{\prime}\right)$. On account of the dynamic nature of $\mathrm{CB}[8]$ host-guest interactions, the dual network is shear sensitive and behaves in a highly viscoelastic manner with increasing angular frequency $(\omega)$. The frequency-dependent viscoelastic moduli clearly identify the elastic behaviour of the dual network with $G^{\prime}>G^{\prime \prime}$ above $\omega=1 \mathrm{rad} \mathrm{s}^{-1}$ (Figure 4a), corroborating the tensile tests at various strain rates (Figure 2a). $G^{\prime}$ of the dual network is constant until $40^{\circ} \mathrm{C}$ and gradually decreases with increasing temperature afterwards (Figure $\mathbf{4 b}$ ). In contrast, $G^{\prime \prime}$ continues to increase and peaks at $c a .45{ }^{\circ} \mathrm{C}$, indicating a soften- 
ing of the dual network, stemming from the increased association/dissociation kinetics of $\mathrm{CB}$ [8] host-guest complexes at higher temperatures. No obvious gel-sol transition is detected throughout the temperature range studied, on account of the covalent crosslinks, which stabilise the dual network structure. Time-temperature superposition (Figure 4c, referenced at $20^{\circ} \mathrm{C}$ ) of the rheological master curve yield an an activation energy $\left(E_{a}\right)$ of $185 \mathrm{~kJ} \mathrm{~mol}^{-1}$, comparable to the typical titin protein unfolding activation energies of 128 $\mathrm{pN} \mathrm{nm}\left(1 \mathrm{~kJ} \mathrm{~mol}^{-1}=1.6 \mathrm{pN} \mathrm{nm}\right.$, thus, $\left.80 \mathrm{~kJ} \mathrm{~mol}^{-1}\right)$. $^{[37]}$ Rapid recovery of its mechanical properties after a largeamplitude oscillatory breakdown is observed at both $20^{\circ} \mathrm{C}$ (see ESI Figure S9a) and $40^{\circ} \mathrm{C}$ (Figure 4d and ESI Figure S10). Upon application of a large-amplitude oscillatory force $\left(\gamma=500 \%, \omega=10 \mathrm{rad} \mathrm{s}^{-1}\right), G^{\prime}$ decreases from 5000 to $600 \mathrm{~Pa}$, resulting in a quasi-liquid state $\left(\tan \delta=G^{\prime \prime} / G^{\prime} \sim\right.$ 2.0). However, when the amplitude decreases $(\gamma=0.5 \%$, $\left.\omega=10 \mathrm{rad} \mathrm{s}^{-1}\right), G^{\prime}$ immediately recovers its initial value at a quasi-solid state $(\tan \delta=0.5)$. Taken together, all of our results successfully demonstrate the biomimetic concept of integrating modular structures held by sacrificial and dynamic CB[8] host-guest complexation into a dual network. These dual network can successfully combine three fundamental mechanical properties, including strength, toughness and elasticity (self-recovery) within one biomimetic system.

In summary, we incorporate force-induced dissociation of dynamic $\mathrm{CB}$ [8] host-guest complexes as a design principle, demonstrating a simple and facile construction of aqueous dual networks, following the structure/function model of titin. These aqueous dual networks are stretchable and tough, showing efficient energy dissipation, and can be fully self-recovered upon relaxation through the reformation of host-guest complexes at room temperature. Both dynamic supramolecular interactions and chemical crosslinks have been readily combined within a single system, yielding a dual network construction represents a powerful and facile strategy for the construction of biomimetic supramolecular materials, holding great promise for myriad applications, including artificial muscles, cartilage replacement and tissue engineering, wearable electronic devices and microactuators.

\section{References}

[1] J. R. Capadona, K. Shanmuganathan, D. J. Tyler, S. J. Rowan, C. Weder, Science 2008, 319, 1370-1374.

[2] A. Miserez, T. Schneberk, C. Sun, F. W. Zok, J. H. Waite, Science 2008, 319, 1816-1819.

[3] X. Li, D. Reinhoudt, M. Crego-Calama, Chem. Soc. Rev. 2007, 36, 1350-1368.

[4] P. Vukusic, J. R. Sambles, Nature 2003, 424, 852-855.

[5] H. Lee, S. M. Dellatore, W. M. Miller, P. B. Messersmith, Science 2007, 318, 426-430.

[6] H. Lee, B. P. Lee, P. B. Messersmith, Nature 2007, 448, 338-341.

[7] A. M. Kushner, Z. Guan, Angew. Chem. Int. Ed. 2011, 50, 90269057.

[8] M. E. Csete, J. C. Doyle, Science 2002, 295, 1664-1669.
[9] S. Labeit, B. Kolmerer, Science 1995, 270, 293-296.

[10] M. S. Kellermayer, S. B. Smith, H. L. Granzier, C. Bustamante, Science 1997, 276, 1112-1116.

[11] M. Rief, M. Gautel, F. Oesterhelt, J. M. Fernandez, H. E. Gaub, Science 1997, 276, 1109-1112.

[12] P. Egan, R. Sinko, P. R. LeDuc, S. Keten, Nat. Commun. 2015, 6 , 7418 .

[13] H. Li, W. A. Linke, A. F. Oberhauser, M. Carrion-Vazquez, J. G. Kerkvliet, H. Lu, P. E. Marszalek, J. M. Fernandez, Nature 2002, 418, 998-1002.

[14] H. Lu, K. Schulten, Biophys. J. 2000, 79, 51-65.

[15] D. L. Guzmán, A. Randall, P. Baldi, Z. Guan, Proc. Natl. Acad. Sci. 2010, 107, 1989-1994.

[16] S. Lv, D. M. Dudek, Y. Cao, M. Balamurali, J. Gosline, H. Li, Nature 2010, 465, 69-73.

[17] J. Fang, A. Mehlich, N. Koga, J. Huang, R. Koga, X. Gao, C. Hu, C. Jin, M. Rief, J. Kast, et al., Nat. Commun. 2013, 4, 2974.

[18] Z. Guan, J. T. Roland, J. Z. Bai, S. X. Ma, T. M. McIntire, M. Nguyen, J. Am. Chem. Soc. 2004, 126, 2058-2065.

[19] A. M. Kushner, V. Gabuchian, E. G. Johnson, Z. Guan, J. Am. Chem. Soc. 2007, 129, 14110-14111.

[20] A. M. Kushner, J. D. Vossler, G. A. Williams, Z. Guan, J. Am. Chem. Soc. 2009, 131, 8766-8768.

[21] M. Guo, L. M. Pitet, H. M. Wyss, M. Vos, P. Y. Dankers, E. Meijer, J. Am. Chem. Soc. 2014, 136, 6969-6977.

[22] R. E. Kieltyka, A. Pape, L. Albertazzi, Y. Nakano, M. M. Bastings, I. K. Voets, P. Y. Dankers, E. Meijer, J. Am. Chem. Soc. 2013, 135 , 11159-11164.

[23] L. Yang, X. Tan, Z. Wang, X. Zhang, Chem. Rev. 2015, 115, 71967239.

[24] C. Li, M. J. Rowland, Y. Shao, T. Cao, C. Chen, H. Jia, X. Zhou, Z. Yang, O. A. Scherman, D. Liu, Adv. Mater. 2015, 27, 3298-3304.

[25] C. S. Tan, J. del Barrio, J. Liu, O. A. Scherman, Polym. Chem. 2015, 6, 7652-7657.

[26] J. Liu, C. S. Y. Tan, Y. Lan, O. A. Scherman, Macromol. Chem. Phys. 2015, 217, 319-332.

[27] S. Fong, S. J. Hamill, M. Proctor, S. M. Freund, G. M. Benian, C. Chothia, M. Bycroft, J. Clarke, J. Mol. Biol. 1996, 264, 624-639.

[28] D. Mozhdehi, J. A. Neal, S. C. Grindy, Y. Cordeau, S. Ayala, N. Holten-Andersen, Z. Guan, Macromolecules 2016, 49, 6310-6321.

[29] J.-Y. Sun, X. Zhao, W. R. Illeperuma, O. Chaudhuri, K. H. Oh, D. J. Mooney, J. J. Vlassak, Z. Suo, Nature 2012, 489, 133-136.

[30] T. L. Sun, T. Kurokawa, S. Kuroda, A. B. Ihsan, T. Akasaki, K. Sato, M. A. Haque, T. Nakajima, J. P. Gong, Nat. Mater. 2013, 12, 932937.

[31] J. P. Gong, Soft Matter 2010, 6, 2583-2590.

[32] F. Luo, T. L. Sun, T. Nakajima, T. Kurokawa, Y. Zhao, K. Sato, A. B. Ihsan, X. Li, H. Guo, J. P. Gong, Adv. Mater. 2015, 27, 2722-2727.

[33] F. Luo, T. L. Sun, T. Nakajima, D. R. King, T. Kurokawa, Y. Zhao, A. B. Ihsan, X. Li, H. Guo, J. P. Gong, Macromolecules 2016, 49, 2750-2760.

[34] A. B. Ihsan, T. L. Sun, T. Kurokawa, S. N. Karobi, T. Nakajima, T. Nonoyama, C. K. Roy, F. Luo, J. P. Gong, Macromolecules 2016, $49,4245-4252$.

[35] P. Lin, S. Ma, X. Wang, F. Zhou, Adv. Mater. 2015, 27, 2054-2059.

[36] M. A. Haque, T. Kurokawa, G. Kamita, J. P. Gong, Macromolecules 2011, 44, 8916-8924.

[37] M. Rief, J. M. Fernandez, H. E. Gaub, Phys. Rev. Lett. 1998, 81, 4764-4767. 